\title{
Multifractality and self-averaging at the many-body localization transition
}

\author{
Andrei Solórzano $\odot,{ }^{1}$ Lea F. Santos $\odot,{ }^{2}$ and E. Jonathan Torres-Herrera $\odot^{3}$ \\ ${ }^{1}$ Tecnologico de Monterrey, Escuela de Ingeniera y Ciencias, Avenida Eugenio Garza Sada 2501, Monterrey, Nuevo Leon, Mexico, 64849 \\ ${ }^{2}$ Department of Physics, Yeshiva University, New York, New York 10016, USA \\ ${ }^{3}$ Instituto de Física, Benemérita Universidad Autónoma de Puebla, Apartado Postal J-48, 72570, Puebla, Mexico
}

(Received 11 February 2021; accepted 23 June 2021; published 30 July 2021)

\begin{abstract}
Finite-size effects have been a major and justifiable source of concern for studies of many-body localization, and several works have been dedicated to the subject. In this Letter, however, we discuss yet another crucial problem that has received much less attention, that of the lack of self-averaging and the consequent danger of reducing the number of random realizations as the system size increases. By taking this into account and considering ensembles with a large number of samples for all system sizes analyzed, we find that the generalized dimensions of the eigenstates of the disordered Heisenberg spin-1/2 chain close to the transition point to localization are described remarkably well by an exact analytical expression derived for the noninteracting Fibonacci lattice, thus providing an additional tool for studies of many-body localization.
\end{abstract}

DOI: 10.1103/PhysRevResearch.3.L032030

The Anderson localization in noninteracting systems has been studied for more than 60 years and it is by now mostly understood [1-3]. Its interacting counterpart, discussed in Refs. [1,4] and analyzed in Refs. [5-11], still presents open questions. It has received enormous theoretical [12-15] and experimental [16-26] attention in the last decade and is often referred to as many-body localization (MBL). There are some parallels between the two cases, but there are also differences, such as the issue of multifractality.

An eigenstate is multifractal when it is extended, but covers only a finite fraction of the available physical space. Multifractality is characterized by the so-called generalized dimension $D_{q}$, for fully delocalized states $D_{q}=1$, for multifractal states $1<D_{q}<0$, and for localized states $D_{q}=0$. In the thermodynamic limit, all eigenstates of one-dimensional (1D) noninteracting systems with uncorrelated random on-site disorder are exponentially localized in configuration space for any disorder strength. It is at higher dimensions that the delocalization-localization transition takes place and this happens at a single critical point, where the eigenstates are multifractal. In contrast, if interactions are added to these systems, the delocalization-localization transition happens already in $1 \mathrm{D}$, and for finite disorder strengths, fractality exists even in the MBL phase. For these interacting systems, it is still under debate whether before the MBL phase there is a single critical point or an extended phase where the eigenstates are multifractal [24,27-41]. In fact, even the very existence of the MBL phase has now gone under debate [42-47]. One of the reasons why it is so hard to settle these disputes is the presence of serious finite-size effects. Recent large-scale

Published by the American Physical Society under the terms of the Creative Commons Attribution 4.0 International license. Further distribution of this work must maintain attribution to the author(s) and the published article's title, journal citation, and DOI. numerical studies $[39,41]$ of the disordered spin-1/2 Heisenberg chain, where Hilbert space dimensions of sizes $\sim 3 \times 10^{6}$ have been reached, did not question the transition to a localized phase, but were not entirely conclusive with respect to the existence of an extended nonergodic phase or a single critical point, although the latter is strongly advocated in Ref. [39].

In this Letter, we consider the same Heisenberg model and emphasize another problem that has not received as much attention as finite-size effects, but is also crucial for studies of disordered systems, that of lack of self-averaging. This issue becomes particularly alarming as the system approaches the transition to the MBL phase $[38,48,49]$. If a quantity is non-self-averaging, the number of samples used in a statistical analysis cannot be reduced as the system size increases [48,50-60]. This reduction is a very common procedure due to the limited computational resources when dealing with exponentially large Hilbert spaces, but it may lead to wrong results. We show that when the disorder strength of the spin model gets larger than the interaction strength and it moves away from the strong chaotic (thermal) regime, the fluctuations of the moments of the energy eigenstates increase as the system size grows, exhibiting a strong lack of self-averaging. Decreasing the number of random realizations in this case may affect the analysis of the structures of the eigenstates, including the results for the generalized dimensions.

The various challenges faced by the numerical studies of the MBL is a great motivator for theoretical works, which, however, have difficulties of their own. The current trend is to focus on phenomenological renormalization group approaches [61-68] that aim at improving our understanding of the MBL transition in 1D systems with quenched randomness, without providing microscopic details. Some of these studies suggest that the transition is characterized by a finite jump of the inverse localization length. Similarly, numerical studies indicate that the generalized dimension jumps at the critical point [39], and a connection between these two jumps was proposed in Ref. [49]. 
Our contribution to those theoretical efforts is to show that an exact analytical expression for the generalized dimensions derived for the 1D noninteracting Fibonacci lattice $[69,70]$ matches surprisingly well our numerical results for the disordered spin-1/2 Heisenberg chain in the vicinity of the MBL critical point. This expression provides an additional tool in the construction of effective models for the MBL transition. Its derivation is based on a renormalization group map of the transfer matrices used to investigate the wave functions of the Fibonacci model [69,70].

Our 1D lattice system has $L$ interacting spin- $1 / 2$ particles subjected to on-site magnetic fields. It is described by the Hamiltonian

$$
H=\sum_{k=1}^{L}\left[S_{k}^{x} S_{k+1}^{x}+S_{k}^{y} S_{k+1}^{y}+S_{k}^{z} S_{k+1}^{z}\right]+\sum_{k=1}^{L} h_{k} S_{k}^{z},
$$

where $S_{k}^{x, y, z}$ are spin- $1 / 2$ operators, the coupling strength was set equal to $1, h_{k}$ are random numbers from a flat distribution in $[-h, h], h$ being the disorder strength, and periodic boundary conditions, $S_{L+1}^{x, y, z}=S_{1}^{x, y, z}$, are imposed. Since $H$ (1) conserves the total spin in the $z$ direction, $\mathcal{S}^{z}=\sum S_{k}^{z}$, we work in the largest subspace corresponding to $\mathcal{S}^{z}=0$, which has dimension $\mathcal{N}=L ! /(L / 2) !^{2}$. The model is integrable when $h=0$ and chaotic, that is, it shows level statistics similar to those from full random matrices [71], when $h_{\text {chaos }} \leqslant$ $h<h_{c}$. The value of $h_{\text {chaos }}$ for the transition from integrability to chaos and of the critical point $h_{c}$ for the transition from delocalization to the MBL phase are not yet known exactly. Our focus here is on the second transition, and for that, some works estimate that $3<h_{c}<4[10,29,72-74]$ and others that $h_{c}>4$ [75-77].

Multifractality and ensemble size. To obtain the generalized dimensions $D_{q}$, we perform scaling analysis of the generalized inverse participation ratios, which are defined as $\operatorname{IPR}_{q}^{\alpha}:=\sum_{k=1}^{\mathcal{N}}\left|\left\langle\phi_{k} \mid \psi_{\alpha}\right\rangle\right|^{2 q}$, where $q$ can take, in principle, any real value, $\left|\psi_{\alpha}\right\rangle$ is an eigenstate of the Hamiltonian (1), and $\left|\phi_{k}\right\rangle$ represents a physically relevant basis. Since we study localization in the configuration space, $\left|\phi_{k}\right\rangle$ is a state where the spins point up or down in the $z$ direction, such as $|\uparrow \downarrow \uparrow \downarrow \cdots\rangle$ [78]. We average the generalized inverse participation ratios $\left\langle\mathrm{IPR}_{q}\right\rangle$ over ensembles with $n$ samples that include $0.02 \mathcal{N}$ eigenstates with energy close to the middle of the spectrum and $n /(0.02 \mathcal{N})$ random realizations, and then extract the generalized dimensions using

$$
\left\langle\operatorname{IPR}_{q}\right\rangle \propto \mathcal{N}^{-(q-1) D_{q}} .
$$

Multifractality holds when $D_{q}$ is a nonlinear function of $q$.

In practice, $D_{q}$ is obtained from the slope of the linear fit of $\ln \left\langle\mathrm{IPR}_{q}\right\rangle$ vs $\ln \mathcal{N}$. In Fig. 1, we show some representative examples of the scaling analysis for different values of $h$ and $q$, and also for ensembles of different sizes $n$, varying from $n=10^{2}$ to $n=3 \times 10^{4}$. The symbols are numerical data and the solid lines are the corresponding fitting curves.

In the chaotic region, for example when $h=1$, the scaling of $\ln \left\langle\mathrm{IPR}_{q}\right\rangle$ with $\ln \mathcal{N}$ is independent of the size of the ensemble, with all points and lines for a given $L$ coinciding and leading to $D_{q} \sim 1$. This is shown in Fig. 1(a) for $q=1.2$ and it holds for all other values of $q$ that we studied, $0.1 \leqslant q \leqslant 3$.
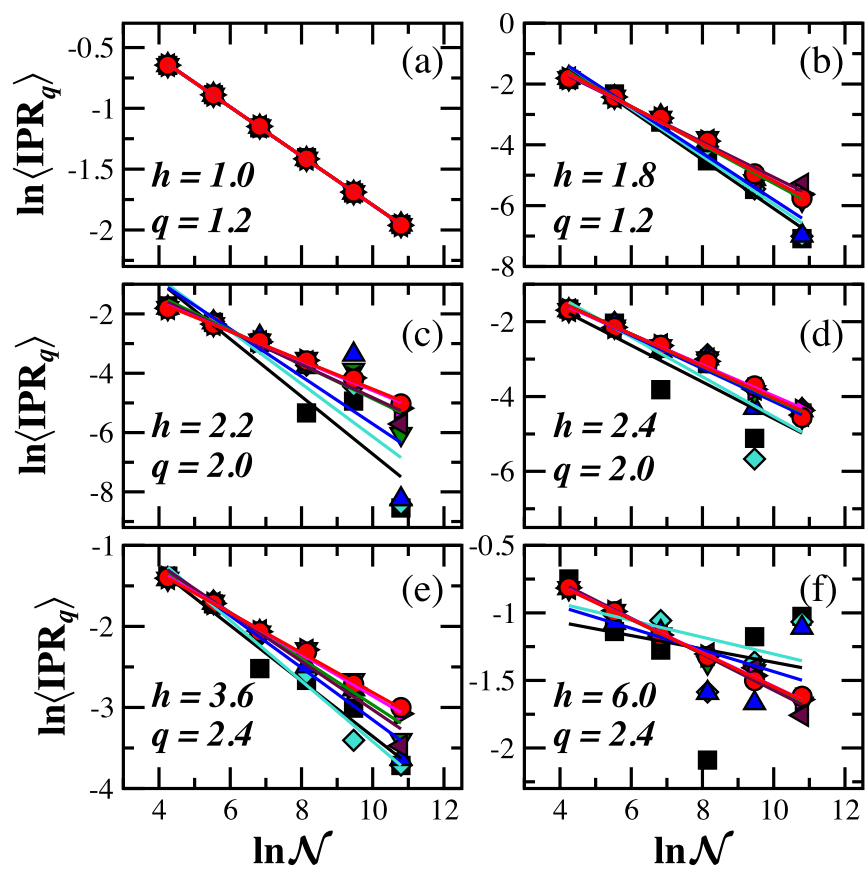

FIG. 1. Scaling of $\ln \left\langle\operatorname{IPR}_{q}\right\rangle$ with respect to the natural logarithm of the Hilbert space dimension $\mathcal{N}$ for various values of $h$ and $q$, as indicated in the panels. Different numbers of samples are considered for the average of $\operatorname{IPR}_{q}: 10^{2}$ (black squares), $5 \times 10^{2}$ (turquoise diamonds), $1 \times 10^{3}$ (blue up triangles), $5 \times 10^{3}$ (green down triangles), $1 \times 10^{4}$ (maroon left triangles), $2 \times 10^{4}$ (magenta right triangles), and $3 \times 10^{4}$ (red circles). The solid lines are the linear fittings for the numerical points and have the same colors as their corresponding points.

In contrast, when $h>1$, the numerical points strongly depend on the number of samples used, as seen from Figs. 1(b)-1(f). Notice that this dependence becomes more evident for the larger system sizes. In the particular cases of Figs. 1(b)-1(e), where $1<h \lesssim h_{c}$, the fittings lead to larger slopes when the ensemble sizes are smaller. For these smaller $n$ 's, the values of $D_{q}$ would get even larger if we would neglect the smallest system sizes when doing the fittings. These results illustrate the danger of reducing the number of samples as the system size increases. By doing so, we can affect the values of $D_{q}$ and therefore the conclusions about the presence or absence of multifractality.

We verify in Figs. 1(b)-1(f) that the convergence of our numerical points happens for ensembles with $n \gtrsim 2 \times 10^{4}$. Indeed, the points for $n=2 \times 10^{4}$ and $n=3 \times 10^{4}$ are nearly indistinguishable, so in all of our subsequent studies, we use $n=3 \times 10^{4}$ for all $L$ 's. It may be, however, that for system sizes larger than the ones considered here, convergence would require even larger ensembles.

Self-averaging. The fluctuations of the values of $\mathrm{IPR}_{q}$ bring us to the discussion of self-averaging. A given quantity $\mathcal{O}$ is self-averaging when its relative variance

$$
R_{\mathcal{O}}=\sigma_{\mathcal{O}}^{2} /\langle\mathcal{O}\rangle^{2},
$$

where $\sigma_{\mathcal{O}}^{2}=\left\langle\mathcal{O}^{2}\right\rangle-\langle\mathcal{O}\rangle^{2}$ decreases as the system size increases [50-58]. This implies that in the thermodynamic limit, 


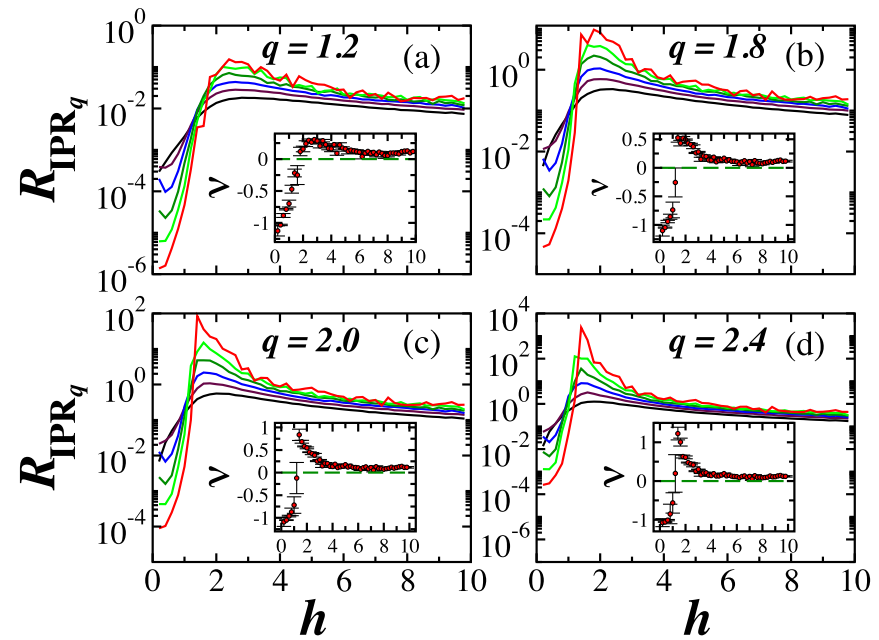

FIG. 2. Relative variance $R_{\mathrm{IPR}_{q}}$ vs disorder strength $h$ for different $q$ 's, as specified in the panels, $3 \times 10^{4}$ statistical data, $\mathcal{N}=$ 70 (black), $\mathcal{N}=256$,(maroon), $\mathcal{N}=924$ (blue), $\mathcal{N}=3432$ (dark green), $\mathcal{N}=12870$ (light green), and $\mathcal{N}=48620$ (red). Insets: Exponent $v$ [Eq. (4)] vs disorder strength $h$. The dashed line marks $v=0$. Error bars are standard errors from a linear fitting.

the result for a single sample agrees with the average over the whole ensemble of samples.

In quantum many-body systems, the eigenstates can spread over the many-body Hilbert space, which is exponentially large in $L$, so we study the scaling of $R_{\mathrm{IPR}_{q}}$ with $\mathcal{N}[48,59]$,

$$
R_{\mathrm{IPR}_{q}} \propto \mathcal{N}^{\nu}
$$

If $v<0$, then $\operatorname{IPR}_{q}$ is self-averaging and one can reduce the number of samples for the average as the system size increases. This cannot be done when $v \sim 0$, and it is even worse in the extreme scenario where $v>0$ and the relative fluctuations increase as the system size grows.

In the main panels of Fig. 2, we show the dependence of $R_{\mathrm{IPR}_{q}}$ on the disorder strength $h$ for different values of $q$ and each line represents one system size. It is clear that deep in the chaotic region, the relative variance decreases as the system size grows, implying self-averaging of $\mathrm{IPR}_{q}$. This is also illustrated in the insets, where $v<0$ for $h_{\text {chaos }} \leqslant h \lesssim 1$, which is consistent with Fig. 1(a), where the scaling of $\mathrm{IPR}_{q}$ does not depend on the number of samples.

There is, however, a turning point at $h \gtrsim 1$, where $v$ suddenly jumps above zero and $R_{\mathrm{IPR}_{q}}$ grows significantly with system size. As seen in Figs. 2(a)-2(d), this is particularly bad in the region preceding the MBL phase, $1 \lesssim h \lesssim 4$. For this range of disorder strength, as the insets indicate, $v>0$ and it reaches large values when $q \gtrsim 2$ [Figs. 2(c) and 2(d)].

For $h \gtrsim 4$, where the system should already be in the MBL phase, the relative variance $R_{\mathrm{IPR}}$ continues to grow with system size, but $v$ is close to zero and the curves for $L=16$ and $L=18$ are not far from each other.

The results in Figs. 1 and 2 show that extra care needs to be taken when performing scaling analysis away from the chaotic region, not only due to finite-size effects, but also due to the lack of self-averaging. No matter how large the system size is, large numbers of samples are required and may even need to be increased as $L$ grows.

One can reduce the fluctuations of the generalized inverse participation ratios by using their logarithm, known as participation Rényi entropies. In fact, using a toy model, it was shown in Ref. [48] that in the MBL phase, $R_{\mathrm{IPR}_{2}}$ grows with system size, while $R_{-\ln I P R_{2}}$ decreases with $L$. However, for $1 \lesssim h \lesssim 4$, even though we observe a reduction of the fluctuations, $\ln \mathrm{IPR}_{q}$ remains non-self-averaging and we still have $v \sim 0$ [79]. We indeed verified that the plots shown in Fig. 1 remain similar if instead of $\ln \left\langle\operatorname{IPR}_{q}\right\rangle$, we use $\left\langle\ln \operatorname{IPR}_{q}\right\rangle$.

Multifractality and analytical expression for $D_{q}$. After taking the necessary precautions for performing the scaling analysis of the generalized inverse participation ratios, as discussed in Fig. 1, we now proceed with the study of how $D_{q}$ depends on $q$ and $h$.

In Refs. [69,70], an exact analytical expression was derived for the structure of the eigenstate at the center of the spectrum of the off-diagonal version of the Fibonacci model in the thermodynamic limit, leading to the generalized dimensions

$$
D_{q}^{\text {Fibonacci }}=\frac{1}{3(q-1) \ln \sigma}\left\{q \ln \left[\lambda\left(h^{2}\right)\right]-\ln \left[\lambda\left(h^{2 q}\right)\right]\right\},
$$

where $\sigma=(\sqrt{5}+1) / 2$ is the golden mean and $\lambda(h)=\{(h+$ $\left.1)^{2}+\left[(h+1)^{4}+4 h^{2}\right]^{1 / 2}\right\} /(2 h)$ is the maximum eigenvalue of the transfer matrix $[69,70]$. For the Fibonacci model, $h$ denotes the ratio between its two hopping constants, which are arranged in a Fibonacci sequence.

In the case of our interacting spin model, we use Eq. (5) as an ansatz. Since in this case, the eigenstates are extended for $h_{\text {chaos }} \leqslant h \lesssim 1$, while Eq. (5) predicts a monotonic decrease of $D_{q}^{\mathrm{Fibonacci}}$ for $h<1$, we compare our results with the expression for $\Theta(h-1) D_{q}^{\mathrm{Fibonacci}}+\Theta(1-h)$, where $\Theta$ is the Heaviside step function. We find that this expression matches the numerical values of $D_{q}$ for the spin chain extremely well for disorder strengths in the vicinity of the critical value, $3<h_{c}<5$.

The inverse participation ratio $\mathrm{IPR}_{2}$ is the most commonly used quantity in studies of localization, so we start by analyzing $D_{2.0}$ and $D_{2.4}$ as a function of the disorder strength. They are shown in Figs. 3(b) and 3(c), respectively. The numerical results agree very well with the analytical expression for $D_{q}^{\text {Fibonacci }}$ for all $h$ 's. However, for smaller $q$ 's, the agreement is not as good, as illustrated in Fig. 3(a), with the same happening for larger $q$ 's, as seen in Fig. 3(d).

We cannot say whether the agreement of the curves for $D_{q}$ vs $h$ with $D_{q}^{\text {Fibonacci }}$ for $q$ away from 2 would improve or get even worse if larger system sizes were considered. If it would improve, that would point to the existence of an extended phase of multifractal eigenstates before the MBL phase and described by the analytical expression of the Fibonacci lattice. Large-scale numerical studies $[39,41]$ indicate that if such a phase exists, it should appear for $h>2$ [41], and it may as well be a single point [39].

We stress, however, that the most relevant and less controversial information provided by Figs. 3(a) and 3(d) is that the numerical points for different values of $q$ 's cross the curve of the analytical expression of $D_{q}^{\text {Fibonacci }}$ at $h \sim h_{c}$ [shaded area in Figs. 3(a) and 3(d)]. This indicates that at least in the vicinity 

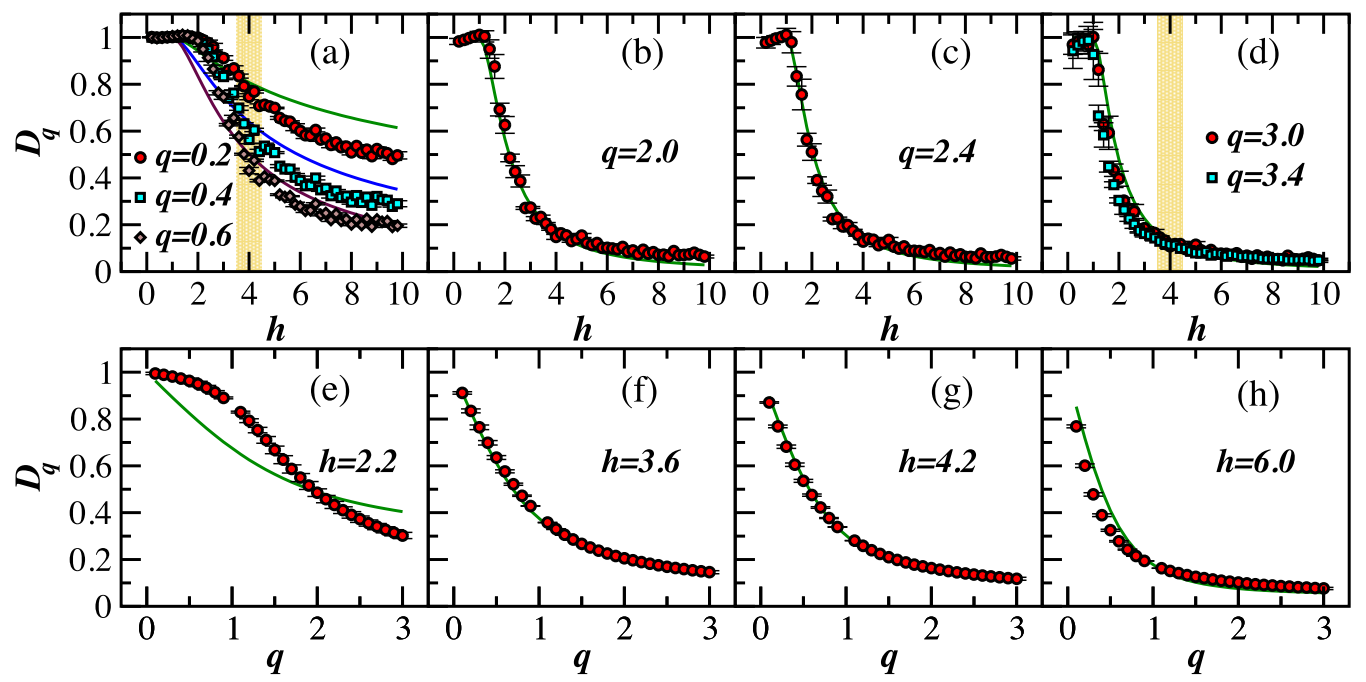

FIG. 3. Top: $D_{q}$ vs disorder strength $h$ for different values of $q$, as indicated in the panels. Bottom: $D_{q}$ vs $q$ for different disorder strengths $h$, as indicated in the panels. Points are numerical results obtained through scaling analysis, averages over an ensemble of $3 \times 10^{4}$ samples. The solid curve gives $\Theta(h-1) D_{q}^{\text {Fibonacci }}+\Theta(1-h)$. Error bars are standard errors from linear fittings. The numerical results in (a) and (d) cross the analytical curve within the shaded vertical area where the critical point should lie.

of (or right at) the critical point, the generalized dimensions of the disordered spin model is indeed extremely well described by Eq. (5).

The bottom panels of Fig. 3 give further support for this observation. There, we plot $D_{q}$ as a function of $q$ for different values of the disorder strength. For $1<h<h_{c}$, as illustrated by Fig. 3(e), there is no good agreement between the numerical points and $D_{q}^{\text {Fibonacci }}$. The same happens for $h>h_{c}$, as seen in Fig. 3(h), although the mismatch in this case is not as large. However, for $h \sim h_{c}$, as shown in Figs. 3(f) and 3(g), the agreement is extremely good.

Conclusions. Our analysis of the disordered spin- $1 / 2$ Heisenberg chain calls attention to the strong lack of selfaveraging of the generalized inverse participation ratios for a range of disorder strengths that precedes the critical point of the MBL transition. This implies that in theoretical and experimental studies of this region, one should not decrease the number of samples as the system size increases. As shown in this Letter, the reduction of the number of random realizations may lead, for example, to inaccurate conclusions regarding multifractality.

We notice also that the logarithm of the generalized inverse participation ratios can be used to reduce fluctuations, but it still does not lead to self-averaging in the vicinity of the critical point.

Our studies indicate a strong relationship between multifractality, $0<D_{q}<1$, and the lack of self-averaging of the generalized inverse participation ratios, $v \geqslant 0$. Multifractality reflects the fragmentation of the Hilbert space [80], and this fragmentation, in turn, leads to the sample-to-sample fluctuations associated with the absence of self-averaging of $\operatorname{IPR}_{q}$. The latter should then hint at the existence of multifractal states.

The comparison between our numerical results for the fractal dimensions of the disordered spin chain and the analytical expression for $D_{q}$ derived for the off-diagonal version of the Fibonacci model shows remarkable agreement in the vicinity of the MBL transition. This connection is useful for theoretical efforts seeking to adequately describe the critical point and may serve as a reference for studies of transport behavior. It should also motivate additional numerical studies to verify whether the agreement holds in a finite region or only at a single critical point.

Acknowledgments. L.F.S. was supported by the NSF Grant No. DMR-1936006. E.J.T.-H. is grateful to LNS-BUAP for their supercomputing facility.
[1] P. W. Anderson, Absence of diffusion in certain random lattices, Phys. Rev. 109, 1492 (1958).

[2] P. A. Lee and T. V. Ramakrishnan, Disordered electronic systems, Rev. Mod. Phys. 57, 287 (1985).

[3] A. Lagendijk, B. van Tiggelen, and D. S. Wiersma, Fifty years of Anderson localization, Phys. Today 62(8), 24 (2009).

[4] L. Fleishman and P. W. Anderson, Interactions and the Anderson transition, Phys. Rev. B 21, 2366 (1980).

[5] B. L. Altshuler, Y. Gefen, A. Kamenev, and L. S. Levitov, Quasiparticle Lifetime in a Finite System: A Nonperturbative Approach, Phys. Rev. Lett. 78, 2803 (1997).
[6] L. F. Santos, G. Rigolin, and C. O. Escobar, Entanglement versus chaos in disordered spin systems, Phys. Rev. A 69, 042304 (2004).

[7] L. F. Santos, M. I. Dykman, M. Shapiro, and F. M. Izrailev, Strong many-particle localization and quantum computing with perpetually coupled qubits, Phys. Rev. A 71, 012317 (2005).

[8] I. V. Gornyi, A. D. Mirlin, and D. G. Polyakov, Interacting Electrons in Disordered Wires: Anderson Localization and Low- $T$ Transport, Phys. Rev. Lett. 95, 206603 (2005).

[9] D. Basko, I. Aleiner, and B. Altshuler, Metal-insulator transition in a weakly interacting many-electron system 
with localized single-particle states, Ann. Phys. 321, 1126 (2006).

[10] V. Oganesyan and D. A. Huse, Localization of interacting fermions at high temperature, Phys. Rev. B 75, 155111 (2007).

[11] F. Dukesz, M. Zilbergerts, and L. F. Santos, Interplay between interaction and (un)correlated disorder in one-dimensional many-particle systems: Delocalization and global entanglement, New J. Phys. 11, 043026 (2009).

[12] R. Nandkishore and D. Huse, Many-body localization and thermalization in quantum statistical mechanics, Annu. Rev. Condens. Matter Phys. 6, 15 (2015).

[13] D. Luitz and Y. B. Lev, The ergodic side of the many-body localization transition, Ann. Phys. (Berlin) 529, 1600350 (2017).

[14] F. Alet and N. Laflorencie, Many-body localization: An introduction and selected topics, C. R. Phys. 19, 498 (2018).

[15] D. A. Abanin, E. Altman, I. Bloch, and M. Serbyn, Colloquium: Many-body localization, thermalization, and entanglement, Rev. Mod. Phys. 91, 021001 (2019).

[16] M. Schreiber, S. S. Hodgman, P. Bordia, H. P. Lüschen, M. H. Fischer, R. Vosk, E. Altman, U. Schneider, and I. Bloch, Observation of many-body localization of interacting fermions in a quasirandom optical lattice, Science 349, 842 (2015).

[17] S. S. Kondov, W. R. McGehee, W. Xu, and B. DeMarco, Disorder-Induced Localization in a Strongly Correlated Atomic Hubbard Gas, Phys. Rev. Lett. 114, 083002 (2015).

[18] J. Smith, A. Lee, P. Richerme, B. Neyenhuis, P. W. Hess, P. Hauke, M. Heyl, D. A. Huse, and C. Monroe, Many-body localization in a quantum simulator with programmable random disorder, Nat. Phys. 12, 907 (2016).

[19] P. Bordia, H. P. Lüschen, S. S. Hodgman, M. Schreiber, I. Bloch, and U. Schneider, Coupling Identical One-Dimensional Many-Body Localized Systems, Phys. Rev. Lett. 116, 140401 (2016).

[20] J.-y. Choi, S. Hild, J. Zeiher, P. Schauss, A. Rubio-Abadal, T. Yefsah, V. Khemani, D. A. Huse, I. Bloch, and C. Gross, Exploring the many-body localization transition in two dimensions, Science 352, 1547 (2016).

[21] P. Bordia, H. Lüschen, U. Schneider, M. Knap, and I. Bloch, Periodically driving a many-body localized quantum system, Nat. Phys. 13, 460 (2017).

[22] P. Bordia, H. Lüschen, S. Scherg, S. Gopalakrishnan, M. Knap, U. Schneider, and I. Bloch, Probing Slow Relaxation and ManyBody Localization in Two-Dimensional Quasiperiodic Systems, Phys. Rev. X 7, 041047 (2017).

[23] A. Lukin, M. Rispoli, R. Schittko, M. E. Tai, A. M. Kaufman, S. Choi, V. Khemani, J. Léonard, and M. Greiner, Probing entanglement in a many-body-localized system, Science 364, 256 (2019).

[24] T. Kohlert, S. Scherg, X. Li, H. P. Lüschen, S. Das Sarma, I. Bloch, and M. Aidelsburger, Observation of Many-Body Localization in a One-Dimensional System with a Single-Particle Mobility Edge, Phys. Rev. Lett. 122, 170403 (2019).

[25] M. Rispoli, A. Lukin, R. Schittko, S. Kim, M. E. Tai, J. Léonard, and M. Greiner, Quantum critical behaviour at the many-body localization transition, Nature (London) 573, 385 (2019).

[26] D. Zhu, S. Johri, N. H. Nguyen, C. Huerta Alderete, K. A. Landsman, N. M. Linke, C. Monroe, and A. Y. Matsuura, Probing many-body localization on a noisy quantum computer, Phys. Rev. A 103, 032606 (2021).
[27] A. D. Luca and A. Scardicchio, Ergodicity breaking in a model showing many-body localization, Europhys. Lett. 101, 37003 (2013).

[28] D. J. Luitz, F. Alet, and N. Laflorencie, Universal Behavior Beyond Multifractality in Quantum Many-Body Systems, Phys. Rev. Lett. 112, 057203 (2014).

[29] D. J. Luitz, N. Laflorencie, and F. Alet, Many-body localization edge in the random-field Heisenberg chain, Phys. Rev. B 91, 081103(R) (2015).

[30] X. Li, S. Ganeshan, J. H. Pixley, and S. Das Sarma, Many-Body Localization and Quantum Nonergodicity in a Model with a Single-Particle Mobility Edge, Phys. Rev. Lett. 115, 186601 (2015).

[31] J. Goold, C. Gogolin, S. R. Clark, J. Eisert, A. Scardicchio, and A. Silva, Total correlations of the diagonal ensemble herald the many-body localization transition, Phys. Rev. B 92, 180202(R) (2015).

[32] E. J. Torres-Herrera and L. F. Santos, Dynamics at the manybody localization transition, Phys. Rev. B 92, 014208 (2015).

[33] X. Li, J. H. Pixley, D.-L. Deng, S. Ganeshan, and S. Das Sarma, Quantum nonergodicity and fermion localization in a system with a single-particle mobility edge, Phys. Rev. B 93, 184204 (2016).

[34] M. Serbyn and J. E. Moore, Spectral statistics across the many-body localization transition, Phys. Rev. B 93, 041424(R) (2016).

[35] W. De Roeck, F. Huveneers, M. Müller, and M. Schiulaz, Absence of many-body mobility edges, Phys. Rev. B 93, 014203 (2016).

[36] W. De Roeck and F. Huveneers, Stability and instability towards delocalization in many-body localization systems, Phys. Rev. B 95, 155129 (2017).

[37] E. J. Torres-Herrera and L. F. Santos, Extended nonergodic states in disordered many-body quantum systems, Ann. Phys. (Berlin) 529, 1600284 (2017).

[38] M. Serbyn, Z. Papić, and D. A. Abanin, Thouless energy and multifractality across the many-body localization transition, Phys. Rev. B 96, 104201 (2017).

[39] N. Macé, F. Alet, and N. Laflorencie, Multifractal Scalings Across the Many-Body Localization Transition, Phys. Rev. Lett. 123, 180601 (2019).

[40] M. Tarzia, Many-body localization transition in Hilbert space, Phys. Rev. B 102, 014208 (2020).

[41] D. J. Luitz, I. M. Khaymovich, and Y. B. Lev, Multifractality and its role in anomalous transport in the disordered XXZ spinchain, SciPost Phys. Core 2, 6 (2020).

[42] J. Šuntajs, J. Bonča, T. Prosen, and L. Vidmar, Quantum chaos challenges many-body localization, Phys. Rev. E 102, 062144 (2020).

[43] D. A. Abanin, J. H. Bardarson, G. De Tomasi, S. Gopalakrishnan, V. Khemani, S. A. Parameswaran, F. Pollmann, A. C. Potter, M. Serbyn, and R. Vasseur, Distinguishing localization from chaos: Challenges in finitesize systems, Ann. Phys. 427, 168415 (2021).

[44] D. J. Luitz and Y. B. Lev, Absence of slow particle transport in the many-body localized phase, Phys. Rev. B 102, 100202(R) (2020).

[45] P. Sierant, D. Delande, and J. Zakrzewski, Thouless Time Analysis of Anderson and Many-Body Localization Transitions, Phys. Rev. Lett. 124, 186601 (2020). 
[46] R. K. Panda, A. Scardicchio, M. Schulz, S. R. Taylor, and M. Žnidarič, Can we study the many-body localisation transition? Europhys. Lett. 128, 67003 (2020).

[47] M. Kiefer-Emmanouilidis, R. Unanyan, M. Fleischhauer, and J. Sirker, Slow delocalization of particles in many-body localized phases, Phys. Rev. B 103, 024203 (2021).

[48] E. J. Torres-Herrera, G. De Tomasi, M. Schiulaz, F. PérezBernal, and L. F. Santos, Self-averaging in many-body quantum systems out of equilibrium: Approach to the localized phase, Phys. Rev. B 102, 094310 (2020).

[49] G. D. Tomasi, I. M. Khaymovich, F. Pollmann, and S. Warzel, Rare thermal bubbles at the many-body localization transition from the Fock space point of view, Phys. Rev. B 104, 024202 (2021).

[50] S. Wiseman and E. Domany, Lack of self-averaging in critical disordered systems, Phys. Rev. E 52, 3469 (1995).

[51] A. Aharony and A. B. Harris, Absence of Self-Averaging and Universal Fluctuations in Random Systems Near Critical Points, Phys. Rev. Lett. 77, 3700 (1996).

[52] S. Wiseman and E. Domany, Finite-Size Scaling and Lack of Self-Averaging in Critical Disordered Systems, Phys. Rev. Lett. 81, 22 (1998).

[53] T. Castellani and A. Cavagna, Spin-glass theory for pedestrians, J. Stat. Mech.: Theory Exp. (2005) P05012.

[54] A. Malakis and N. G. Fytas, Lack of self-averaging of the specific heat in the three-dimensional random-field Ising model, Phys. Rev. E 73, 016109 (2006).

[55] S. Roy and S. M. Bhattacharjee, Is small-world network disordered? Phys. Lett. A 352, 13 (2006).

[56] C. Monthus, Random walks and polymers in the presence of quenched disorder, Lett. Math. Phys. 78, 207 (2006).

[57] A. Efrat and M. Schwartz, Lack of self-averaging in random systems - Liability or asset? Phys. A: Stat. Mech. Appl. 414, 137 (2014).

[58] M. Łobejko, J. Dajka, and J. Łuczka, Self-averaging of random quantum dynamics, Phys. Rev. A 98, 022111 (2018).

[59] M. Schiulaz, E. J. Torres-Herrera, F. Pérez-Bernal, and L. F. Santos, Self-averaging in many-body quantum systems out of equilibrium: Chaotic systems, Phys. Rev. B 101, 174312 (2020).

[60] E. J. Torres-Herrera, I. Vallejo-Fabila, A. J. Martínez-Mendoza, and L. F. Santos, Self-averaging in many-body quantum systems out of equilibrium: Time dependence of distributions, Phys. Rev. E 102, 062126 (2020).

[61] R. Vosk, D. A. Huse, and E. Altman, Theory of the Many-Body Localization Transition in One-Dimensional Systems, Phys. Rev. X 5, 031032 (2015).

[62] L. Zhang, B. Zhao, T. Devakul, and D. A. Huse, Many-body localization phase transition: A simplified strong-randomness approximate renormalization group, Phys. Rev. B 93, 224201 (2016).

[63] P. T. Dumitrescu, R. Vasseur, and A. C. Potter, Scaling Theory of Entanglement at the Many-Body Localization Transition, Phys. Rev. Lett. 119, 110604 (2017).
[64] T. Thiery, F. Huveneers, M. Müller, and W. De Roeck, ManyBody Delocalization as A Quantum Avalanche, Phys. Rev. Lett. 121, 140601 (2018).

[65] A. Goremykina, R. Vasseur, and M. Serbyn, Analytically Solvable Renormalization Group for the Many-Body Localization Transition, Phys. Rev. Lett. 122, 040601 (2019).

[66] P. T. Dumitrescu, A. Goremykina, S. A. Parameswaran, M. Serbyn, and R. Vasseur, Kosterlitz-thouless scaling at manybody localization phase transitions, Phys. Rev. B 99, 094205 (2019).

[67] A. Morningstar and D. A. Huse, Renormalization-group study of the many-body localization transition in one dimension, Phys. Rev. B 99, 224205 (2019).

[68] A. Morningstar, D. A. Huse, and J. Z. Imbrie, Many-body localization near the critical point, Phys. Rev. B 102, 125134 (2020).

[69] T. Fujiwara, M. Kohmoto, and T. Tokihiro, Multifractal wave functions on a Fibonacci lattice, Phys. Rev. B 40, 7413 (1989).

[70] H. Hiramoto and M. Kohmoto, Electronic spectral and wavefunction properties of one-dimensional quasiperiodic systems: A scaling approach, Int. J. Mod. Phys. B 06, 281 (1992).

[71] T. Guhr, A. Mueller-Gröeling, and H. A. Weidenmüller, Random matrix theories in quantum physics: Common concepts, Phys. Rep. 299, 189 (1998).

[72] A. Pal and D. A. Huse, Many-body localization phase transition, Phys. Rev. B 82, 174411 (2010).

[73] T. C. Berkelbach and D. R. Reichman, Conductivity of disordered quantum lattice models at infinite temperature: Many-body localization, Phys. Rev. B 81, 224429 (2010).

[74] J. A. Kjäll, J. H. Bardarson, and F. Pollmann, Many-Body Localization in a Disordered Quantum Ising Chain, Phys. Rev. Lett. 113, 107204 (2014).

[75] T. Devakul and R. R. Singh, Early Breakdown of Area-Law Entanglement at the Many-Body Delocalization Transition, Phys. Rev. Lett. 115, 187201 (2015).

[76] E. V. H. Doggen, F. Schindler, K. S. Tikhonov, A. D. Mirlin, T. Neupert, D. G. Polyakov, and I. V. Gornyi, Many-body localization and delocalization in large quantum chains, Phys. Rev. B 98, 174202 (2018).

[77] P. Sierant, M. Lewenstein, and J. Zakrzewski, Polynomially Filtered Exact Diagonalization Approach to Many-Body Localization, Phys. Rev. Lett. 125, 156601 (2020).

[78] The inverse participation ratio for eigenstates written in the basis of the Anderson localized states is also non-self-averaging in the vicinity of the transition point to the MBL phase.

[79] See Supplemental Material at http://link.aps.org/supplemental/ 10.1103/PhysRevResearch.3.L032030 for the application of a logarithmic transformation to the generalized inverse participation ratios of the energy eigenstates, this is not enough to achieve self-averaging, specially around the critical point.

[80] F. Pietracaprina and N. Laflorencie, Hilbert-space fragmentation, multifractality, and many-body localization, Ann. Phys., 168502 (2021). 